\title{
La ponctuation dans quelques imprimés de Galliot du Pré
}

\author{
The punctuation in Galliot du Pré's printed works
}

Huei-Chen Li

Université de Srasbourg, France

\begin{abstract}
Résumé : D'après Annie Charon-Parent, Galliot du Pré, libraire-éditeur du XVle siècle, publie 330 éditions durant sa carrière, entre 1521 et 1561. Elles touchent essentiellement le droit, la littérature, l'histoire, la religion, les sciences, ainsi que la géographie. Comme nous le savons bien, la ponctuation a pour principale fonction de rendre le texte plus intelligible et d'aider le lecteur à repérer le sens des phrases et la structure du texte: cette fonction de rendre le texte clair répond parfaitement au goût des humanistes. Pourtant, face à l'anarchie et à la variabilité de la ponctuation médiévale, la pratique de la ponctuation renaissante, avec l'avènement de l'imprimerie, est-elle plus stable que celle du Moyen Âge ? Respecte-elle les règles recommandées par les traités médiévaux ou ceux de l'époque ? Notre étude, basée sur trois imprimés de Galliot du Pré parus entre 1522 et 1530, tous conservés à la BNF (Rés. Y2-72; Rés. Y2-31 ; Rés. Y2-74), vise à présenter les pratiques de chaque imprimé afin de mettre au clair le sous-système de Galliot du Pré, un homme de la Renaissance.
\end{abstract}

Mots-clés : Galliot du Pré, ponctuation à la Renaissance, Rés. Y2-72 (Ysaie le Triste), Rés. Y2-31 (Roman de Perceforest), Rés. Y2-74 (Perceval le Galloys en prose).

Abstract: According to Annie Charon-Parent, Galliot du Pré, bookseller-publisher of the $16^{\text {th }}$ century, published 330 editions during his career between 1521-1561. These editions relate essentially to the law, the literature, the history, the religion, the sciences, and the geography. As we know that the primary function of punctuation is to make the text clearer and more understandable, it also helps the silent reader to identify the sense of sentences and the structure of the text, this function of clarifying the text suits the humanist needs. Nevertheless, in comparison with the variability of the medieval punctuation, with the advent of the printing press, is the punctuation of the Renaissance more stable than the medieval punctuation? Does the Renaissance punctuation respect the rules recommended by medieval and Renaissance treaties? Our study, based on three Galliot du Pré's printed works between 1522-1530, kept all in the National Library of France (Res. Y2-72; Res. Y2-31; Res. Y2-74), aims at presenting the practices of each printed work in order to clear up Galliot du Pré's sub-system.

Keywords: Galliot du Pré, punctuation of the sixteenth century, Ysaie le Triste, Perceforest, Perceval le Galloys in prose.

\section{Introduction}

Selon Nina Catach (1994: 11-32), les Grecs d'Alexandrie ont très tôt utilisé des signes de ponctuation dans les manuscrits en scriptio continua : ils ont distingué à la base le point en haut, le point médian et le point en bas pour marquer respectivement les ponctuations forte, moyenne et faible. Toutefois, I' histoire nous apprend que, malgré les efforts des grammairiens du Moyen Âge qui cherchent à fixer de manière répétitive les valeurs des signes, les théories sont loin d'être conformes à la pratique, 
car les formes des signes peuvent rester les mêmes, alors que leurs appellations et leurs valeurs linguistiques ont changél.

En 1471, le premier traité de ponctuation, imprimé en France par Guillaume Ficheł et Jean Heynlin sur les presses de la Sorbonne, s'intitule Compendiosus dialogus de arte punctandi2: il se trouve à la fin de l'Orthographia de l'humaniste italien Gasparino Barzizza. S'inspirant de la Doctrina punctandi de Gasparino Barzizza, ce traité, qui cherche à concilier les usages anciens et modernes, présente une liste de huit signes avec l'explication de leurs fonctions ${ }^{3}$. Cela prouve qu'au début de l'imprimerie, soucieux d'améliorer la clarté du texte, les premiers imprimeurs portent un grand intérêt aux problèmes de la ponctuation.

En 1529, Jacques Lefèvre d'Ełaples et Geoffroy Tory4 préconisent chacun leur nouveau système, encore plus riche et hardi que le traité précité. Toutes ces tentatives théoriques de normaliser le système graphique aux XVe et XVle siècles nous conduisent à nous interroger sur les pratiques de la ponctuation dans les imprimés du début du XVle siècle, publiés par Galliot du Pré (1491-1561)5.

Nous commencerons par présenter notre corpus, basé sur trois imprimés de Gallioł du Pré, tous conservés à la BNF (Rés. Y2-72; Rés. Y2-31; Rés. Y2-74), ensuite nous procéderons à l'analyse comparative des pratiques de chaque imprimé afin de mettre au clair le sous-système de Galliot du Pré, libraire-éditeur du XVle siècle.

\section{Présentation du corpus}

Notre corpus comprend trois imprimés gothiques conservés à la Bibliothèque Nationale de France. Ils sont tous imprimés pour le libraire-éditeur Galliot du Pré entre 1522 et 1530. Soulignons qu'ils contiennent tous des textes relevant du genre du roman de chevalerie en prose 6 , constituant un ensemble narratif assez complet pour permettre d'étudier l'agencement du récit. Le corpus est présenté grosso modo dans le tableau ci-dessous:

\footnotetext{
${ }^{1} \mathrm{~N}$. Catach a remarqué l'écart entre les théories et la pratique : surtout, le même signe peut changer d'appellation d'un jour à l'autre, selon les traités et les périodes. Par exemple, le colon au Moyen Âge correspond à une ponctuation moyenne, et le comma joue le rôle de la ponctuation faible : pourtant, au XVle siècle, dans le traité d'Etienne Dolet, à l'inverse des grammaires médiévales, c'est le comma qui assume la valeur de ponctuation moyenne, et la ponctuation faible porte un autre nom, à savoir le point à queve, virgule, ou incisum. Pour plus détails, voir également C. Buridant (1980: 13-53); N. Catach (1968: 295-313; $1988: 33-47)$; M. Hubert (1972: 55-72) ; A. Lavrentiev (2000: $23-35 ; 2011: 31-$ $56 ; 2016: 39-62)$.

2 Selon I'hypothèse d' É. Beltran (1985: 286-287), c'est Guillaume Fichet qui doit être l'auteur de ce traité, tandis que la tradition attribue l'ouvrage à Jean Heynlin.

3 D'après A. Lavrentiev (2011 : 33-36), le Maître présente huit signes : la virgula, le comma, le colon, le periodus, le punctus interrogativus, les parenthesis, le gemipunctus, et le semipunctus.

${ }^{4}$ Selon N. Catach (1994 : 30), Geoffroy Tory, dans son Champ Fleury, présente onze signes, contre sept chez Etienne Dolet.

5 L'année de mort de Galliot du Pré d'après les historiens du livre reste discutable. A. Charon-Parent (1988 : 209) suppose que Galliot du Pré est né probablement vers 1491, et mort en 1561, or, selon P. Delalain (1891: 14), Galliot du Pré est décédé en 1560: cette date de mort est fournie par la transcription d'une pierre votive conservée au Musée de Cluny.

${ }^{6}$ Annie Charon-Parent (1988 : 214) divise l'activité éditoriale de Galliot du Pré en trois grandes périodes: les années 1528-1530, période florissante, où il privilégie la littérature et l'histoire; l'année 1539, où il publie l'ordonnance de Villers-Cotterêts; les années 1544-1545, où il s'intéresse de nouveau à la production d'ouvrages juridiques.
} 


\begin{tabular}{|c|c|c|}
\hline Description des imprimés & Corpus & $\begin{array}{l}\text { Combinaisons } \\
\text { de signes }^{7}\end{array}$ \\
\hline $\begin{array}{l}\text { Cote : Bibliothèque Nationale de France, Rés. Y2-72 } \\
\text { Contenu : Ysaie le Triste filz Tristan de leonois/ jadis } \\
\text { chevalier de la table ronde/ et de la royne Izeut de } \\
\text { Cornouaille [...] } \\
\text { Date d'édition : } 1522 \\
\text { Libraire-éditeur : Galliot du Pré }\end{array}$ & $\begin{array}{l}\text { Chapitres I et } \\
\text { II : fol. I r-2 r }\end{array}$ & $\begin{array}{l}251 \\
\text { ponctuations } \\
\text { de } 11 \text { sortes }\end{array}$ \\
\hline $\begin{array}{l}\text { Cote : Bibliothèque Nationale de France, Rés. Y2-31 } \\
\text { Contenu : Le quart volume des Anciennes Croniques } \\
\text { Dangleterre/ Faictz et Gestes dy roy Perceforest/ et } \\
\text { des chevaliers du Franc Palais, imrprimé à Paris, par } \\
\text { Nicolas Cousteau pour Galliot du Pré. } \\
\text { Date : } 1528 \\
\text { Libraire-éditeur : Galliot du Pré }\end{array}$ & $\begin{array}{l}\text { Chapitre II: } \\
\text { fol. } 12 \mathrm{r}-13 \mathrm{r}\end{array}$ & $\begin{array}{c}233 \\
\text { ponctuations } \\
\text { de } 11 \text { sortes }\end{array}$ \\
\hline $\begin{array}{l}\text { Cote : Bibliothèque Nationale de France, Rés. Y2-74 } \\
\text { Contenu: Tresplaisante et recreative hystoire du } \\
\text { trespreulx et vaillant Chevallier Perceval le galloys } \\
\text { Jadis chveallier de la Table ronde [...] } \\
\text { Date: } 1530 \\
\text { Libraires-éditeurs: Jehan Longis. Jehan sainct Denys/ } \\
\text { et Galliot du Pré }\end{array}$ & $\begin{array}{l}\text { Chapitre I: } \\
\text { fol. } 2 r-3 v\end{array}$ & $\begin{array}{c}185 \\
\text { ponctuations } \\
\text { de } 11 \text { sortes }\end{array}$ \\
\hline
\end{tabular}

\section{Principes d'analyse}

Nous entendons par ponctuation un système graphique qui participe à l'organisation du texte écrit. Pour faciliter l'analyse, en nous inspirant de la classification de Nina Catach8 (1994: 7-8), nous distinguerons trois niveaux de ponctuation: le niveau textuel, le niveau inter-propositionnel 9 et le niveau intrapropositionnel, qui comprend le niveau des syntagmes et le niveau des mots.

\subsection{Analyse de l'édition imprimée d'Ysaie le Triste (251 cas)}

\subsubsection{Au niveau textuel ( 7 cas, $2.7 \%$ )}

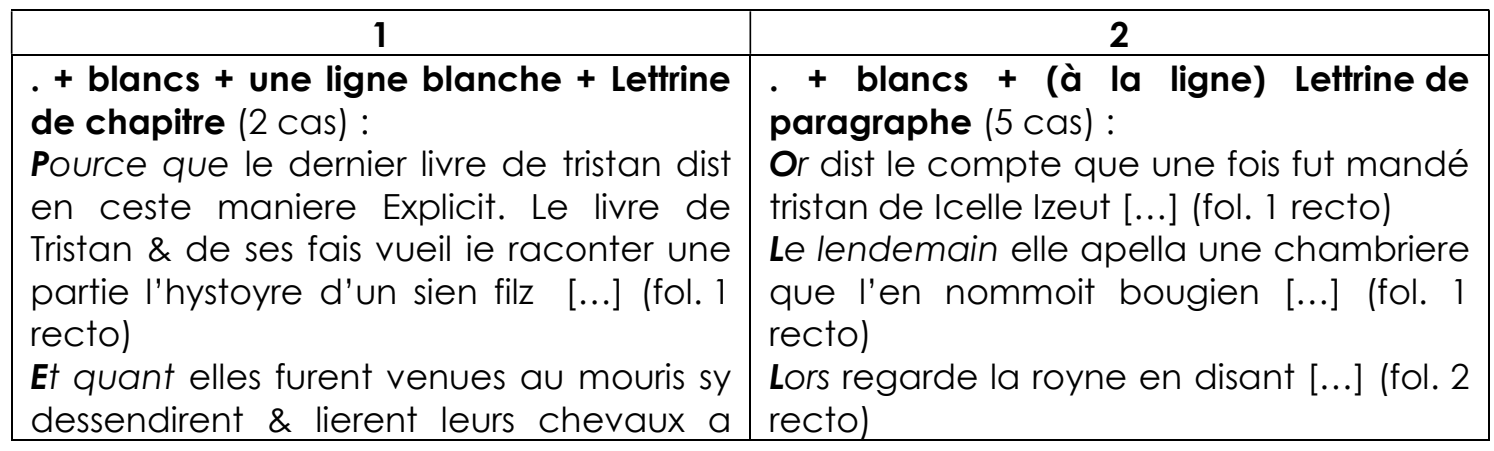

\footnotetext{
7 Nous emploierons dans notre analyse le symbole " $\varnothing$ " pour signifier l'absence de signe, " . " pour le signe point, " / " pour le signe barre oblique, "I" " pour le signe pied-de-mouche, la lettre "M " pour majuscule et la lettre «m» pour minuscule. Notre étude inclura la combinatoire "signe zéro + Majuscule "n au niveau inter-propostionnel.

${ }^{8} \mathrm{~N}$. Catach distingue trois types de ponctuation : la ponctuation des mots, la ponctuation syntaxique et communicative, et la ponctuation du texte ou mise en page.

9 Nous suivons la terminologie de C. Marchello-Nizia (1978 b: 33) en évitant d'employer le mot "phrase ", compte tenu de l'ambiguïté notionnelle de ce mot.
} 
ung chesne [...] (fol. 2 verso)

Or dist le conte que la royne dist a L'hermite [...] (fol. 2 recto)

Incontient aprés la mort Tristan/ et Izeut/ [...] (fol. 2 recto)

Les chapitres I et II d'Ysaie le Triste sont composés de sept « unités de lecture $»^{10}$ au niveau textuel :

- 2 lettrines de chapitre occupant sept lignes de haut.

- 5 lettrines de paragraphe qui occupent tantôt trois lignes de haut, tantôt quatre lignes de haut.

Cette pratique du découpage du texte par le biais des lettrines, appelées litterae notabiliores en latin (Ouy, 1979 : 70-71), est particulièrement courante dans les manuscrits médiévaux. Cependant, malgré l'invention de la typographie qui commence à réduire progressivement le nombre des lettrines au XVIe siècle, par peur de bouleverser radicalement l'habitude de lecture, les typographes continuent à élaborer les éditions suivant le modèle des manuscrits anciens.

En ce qui concerne les éléments qui se trouvent à l'entrée du chapitre ou des paragraphes, il n'est pas étonnant que les struments temporels (6 cas) s'imposent dans un contexte narratif, car l'action des personnages s'inscrit dans une temporalité. Remarquons qu'un cas exprimant la causalité (Pource que) se rencontre dans notre tableau.

\subsubsection{Au niveau inter-propositionnel (156 cas, $62 \%$ )}

\begin{tabular}{|c|c|c|c|c|c|c|}
\hline $\mathbf{3}$ & $\mathbf{4}$ & $\mathbf{5}$ & $\mathbf{6}$ & $\mathbf{7}$ & $\mathbf{8}$ & $\mathbf{9}$ \\
\hline $\mathbf{q}+\mathbf{M}$ & $++\mathbf{M}$ & $:+\mathbf{M}$ & $/+\mathbf{M}$ &.$+\mathbf{m}$ & $:+\mathbf{m}$ & $/+\mathbf{m}$ \\
$(2$ cas $)$ & $(34$ cas $)$ & $(1$ cas $)$ & $(4$ cas $)$ & $(3$ cas $)$ & $(27$ cas $)$ & $(85$ cas $)$ \\
\hline
\end{tabular}

\section{Les combinatoires avec majuscule ${ }^{11}$}

La combinatoire 3 signale le début du livre et du chapitre :

(1) If Cy commence ${ }^{12}$ la noble hystoire de ysaye le triste qui fut filz illegittime du vaillant Tristan de lyonnoys Chevalier de la table Ronde. (fol. 1 recto)

(2) If Comment la royne Izeut acoucha a l'entree d'un boys de Isaye le triste le quel fut baptisé \& nourry d'un hermite. (fol. 1 verso)

Dans l'exemple (1), l'embrayeur "cy » participe à la structuration du texte, car il indique le titre du livre, alors que l'exemple (2) commençant par "comment» nous présente globalement le contenu du chapitre.

Les combinatoires 4 à 6 délimitent assez souvent des propositions non dépendantes ou des séquences corrélatives "subordonnée-principale»

\footnotetext{
10 J'emprunte cette expression à C. Marchello-Nizia (1978 a : 44).

11 A. Doppagne (1991: 11-24) distingue deux fonctions de la majuscule, à savoir la fonction démarcative et la fonction distinctive.

12 Nous reproduisons fidèlement la ponctuation médiévale et la segmentation graphique ou agglutination-déglutination graphique que N. Andrieux-Reix appelle séquence graphique (1997 : 290). Soulignons que pour des raisons de lisibilité, nous avons décidé de développer les abréviations en toutes lettres, en les soulignant.
} 
commençant soit par des sujets nominaux ou pronominaux, soit par des struments d'ordre divers:

(3). En tel guise demoura lzeut ung moys moult malade \& pale/ [...] (fol. 2 recto)

(4) . Car sa voulenté est la mienne/ \& la mienne est la sienne. [...] (fol. 2 verso)

(5). Mais pource que les rommans ne vouloient parler de la villennie qu'il conceupt contre l'onneur d'icelle sy m'en tais. Ou par adventure que celuy qui fist le livre ne le sçavoit mye : \& pour ce que ie vueil que riens n'en soit revelé : Vous pense a dire que l'honneur y fust [...] (fol. 1 recto)

(6) . Je me fye moult a toy/ [...] (fol. 1 recto)

(7) / Izeut spandent mettoit les heures en escript que tristan gisoit avec elle [...] (fol. 1 recto)

(8) / Je ne vueil fait il nul bien ains le voys deffier comme faulx (fol. 1 verso)

(9). Quant ce vint au bout de sept moys elle sentit ses maulx/ \& se doubta moult qu'elle ne fust aperceue. [...] (fol. 1 recto)

(10) . Lors advisa que le roy marc devoit aler vers ung tournoy qui devoit estre devant l'estrange passaige. Sy passa sa chambriere et luy dist/ [...] (fol. 1 recto-1 verso)

Par ailleurs, on constate que les combinatoires 4 et 6 marquent aussi quelquefois le début du discours direct, le changement de locuteur et le retour à la narration :

(11) A tant le preudhomme s'aproche d'elle/ \& elle commence a dire. Sire toutes les besoingnes dont je me suis meffaicte vers le roy Marc mon mary [...] (fol. 1 recto)

(12) Or dist le conte que la royne dist a L'hermite/ sire donnés a mon filz tel nom que bon vous semble/ Voulentiers fait il/ aussi ie le garderay tant qu'il puist porter armes/ [...] (fol. 2 recto)

(13) . Dame fait il/ voulentiers. Vous avez a nom Izeut/ \& son pere Tristan/ Isaye le triste. Certes fait elle/ ie l'otroye/ car assez enssieult mon nom/ \& le nom de son pere. Atant bouta L'ermite l'enfant en la fontaine/ au nom du pere/ du filz/ \& du saint esperit [...] (fol. 2 recto)

(14) \& dist lzeut comment est vostre nom ie suys fait il nommé Lucas le bouteillier/ \& que voulez vous au roy Marc/ Je ne vueil fait il nul bien ains le voys deffier comme faulx : \& traitre/ [...] (fol. 1 verso)

\section{Les combinatoires avec minuscule}

La combinatoire 8 se rencontre de manière récurrente devant les propositions commençant par le coordonnant « et» (16 cas) :

(15) : \& le roy estoit allé iover a ung sien chastel loing de tintageul a .xi. lieves. [...] (fol. 1 recto)

(16) : \& lors il se partit tout monté \& armé : quant il eult prins congié ycelle iournee a l'heure de nonne vint le roy Marc/ Izeut spandent mettoit les heures en escript que tristan gisoit avec elle : \& aussy faisoyt elle du roy son mary. [...] (fol. 1 recto) 
(17) : \& quant il fut venu sy regarda en l'eure la royne/ \& se agenoula en disant [...] (fol. 1 verso)

Dans la plupart des cas relevant de la combinatoire 8, comme l'a constaté A. Lavrentiev (2011 : 35) à propos du compendiosus dialogus de arte punctandi, le comma s'emploie après les expressions contenant un verbe, mais ayant un sens incomplet, c'est pourquoi il balise parfois des propositions ayant des éléments communs:

(18) E lendemain elle apella une chambriere que l'en nommoit bougien : \& luy dist en briefves parolles.

Il ne faut pas perdre de vue que la combinatoire 7 apparaît deux fois pour signaler l'alternance des interlocuteurs au cours de la conversation :

(19) or nous dictes ou allés vous. ie voys dist il a Tintageul parler au roy Marc/ [...] (fol. 1 verso)

(20) Lors (l'ermite) regarde la royne en disant/ trop vous estes meffaite avec ung si vaillant homme comme est le roy Marc/ \& que tant tenoit de terriennes honneurs vous meffeistes. Car il vous avoit en grant foy espousee. helas sire fait elle aultrement ne peult estre [...] (fol. 2 recto)

La combinatoire 9 délimite aussi bien des propositions non dépendantes que des propositions principales postposées aux subordonnées:

(21) Quant la mere veyt l'enfant qui sy bel estoit/ sy le baise \& acolle/ \& adonc apelle sa chambriere en disant. [...] (fol. 1 verso)

(22) Je me fye moult en toy/ ie suis grosse de Tristan de Leonnoys/ sy vueil que tu me aydes a me conseillier/ \& soys certaine que s'il en est nouvelles autant te voudroyt estre morte. [...] (fol. 1 recto)

(23) Quant ce vint ung peu aprés/ elle se print a acoucher d'un enfant masle [...] (fol. 1 verso)

(24) / mais ie n'em vueil point faire long conte/ car ie vueil emprendre le moins/ sur le livre Tristan que ie puis/ neautmoingz y ayié prins mon theusme/ \& ay assez raison/ car le commencement du filz doibt venir du pere. [...] (fol. 2 recto)

De surcroît, la combinatoire 9 démarque assez régulièrement des subordonnées :

(25) \& elle respondit/ que ce vouloit elle bien/ affin que nouvelles n'en soit a court [...] (fol. 2 recto)

(26) Car les amours de Tristan avoient commencé par la vertu d'un beuvraige/ que la femme du roy Argius D'irlande mere de Izeut bailla a gomorail/ \& a Bougien/ [...] (fol. 2 recto)

(27) Je ne vueil fait il nul bien ains le voys deffier comme faulx : \& traitre/ qui a navré Tristan de lyonnois en traison [...] (fol. 1 verso)

\subsubsection{Au niveau intra-propositionnel ( 88 cas, $35 \%$ )}

\begin{tabular}{|c|c|c|c|c|c|}
\hline 4 & 6 & 8 & 9 & 10 & 11 \\
\hline$\dot{+}+M$ & $/+M$ & $:+m$ & $/+m$ &. chiffre romain. & $/ /$ en fin de ligne \\
$(2$ cas $)$ & $(8$ cas $)$ & $(6$ cas $)$ & $(39$ cas $)$ & ( 3 cas $)$ & (30) \\
\hline
\end{tabular}


D'après notre enquête, on constate que les combinatoires 4, 6, 8 et 9 ponctuent les syntagmes nominaux dans une série énumérative :

(28) dont il avoit occis Huneson l'amy/ Morgeam sy assambla partie des Chevaliers de la table Ronde/ Behourd de gannes. Et Hector des mares/ Bliomberis de gannes/ Percheval de galles/ Brun le noir/ Gaheriet/ \& Gaharet/ \& monseigneur Gauvain/ \& le roy Baudemagus/le roy Carados/ \& le roy des cent chevaliers/ et le roy de norgalles/ et Lucquan le boutillier/ et segremors le desraes/ Hector le filz ares/ \& Agrevain/ \& yvain : le filz au roy Uerin/ et Lionnel : et kelly le senechal : \& Dodinel le sauvaige [...] (fol. 2 recto)

Il est à noter que la combinatoire 9 se rencontre aussi dans l'énumération de syntagmes de même fonction syntaxique:

(29) L'ermite l'enfant en la fontaine/ au nom du pere/ du filz/ \& du saint esperit [...] (fol. 2 recto)

Les syntagmes prépositionnels sont épisodiquement précédés du signe «barre oblique $॥$ :

(30) car ie vueil emprendre le moins/ sur le livre Tristan que ie puis [...] (fol. 2 recto)

(31) le roy Marc avoit navré Tristan/ en trahison donc mort s'en estoit ensuivye [...] (fol. 2 recto)

Nous repérons trois fois la combinatoire 10 pour marquer le chiffre romain :

(32) le roy estoit allé iover a ung sien chastel loing de tintageul.xi. lieves [...] (fol. 1 recto)

(33) non mye sy brief qu'il n'y eult .xxx. feulletz [...] (fol. 1 verso)

A. Lavrentiev (2011 : 35-36) mentionne que dans le compendiosus dialogus de arte punctandi, il existe un signe appelé gemipunctus (deux points successifs disposés horizontalement) servant à mettre en valeur les noms de personnes, de lieux ou de titres de noblesse. Ce signe peut équivaloir aux points symétriques encadrant les chiffres romains dans les manuscrits médiévaux.

La combinatoire 11 concerne plutôt la ponctuation de mot, puisqu'elle marque la coupure de mots en fin de ligne:

(34) : \& y soit petit a l'u//ne partie/ (fol. 1 recto)

(35) des qu'il apor//ta une espee (fol. 2 recto)

Les lignes, limitées par des espaces, ne peuvent pas coucher librement tous les mots entiers, notamment les mots polysyllabes. II arrive que les mots plurisyllabes tombent en fin de ligne : en l'occurrence, l'éditeur a recours aux deux traits obliques pour indiquer que les mots chevauchent sur deux lignes. II est notable qu'en fin de ligne, l'édition de 1522 découpe les mots en respectant la loi de syllabation.

G. Ouy (1979: 69) nous informe que Chez G. Barzizza, un signe ayant la forme d'un tiret horizontal s'appelle la virgule couchée (virgula jacens), et qu'il assume la 
fonction de coupure de mots en fin de ligne ${ }^{13}$. Cette fonction peut correspondre à celle du semipunctus proposé par le traité de Guillaume Fichet et de Jean Heynlin.

Enfin, il importe de signaler la présence générale de la majuscule à l'initiale des noms propres dans cet imprimé (Izeut, Tristan, Bougien, Marc, Hector, Gauvain, Carados, Cornuaille, Leonnoys, Isaye, Lancelot, etc.) : il n'est pas exclu cependant que l'on emploie la minuscule pour noter les noms propres (marc, ysaye, tristan, yvain, lyonnoys, gannes, etc.). Précisons que lorsque les noms de lieux sont précédés de la préposition « de », que l'article soit présent ou non (des mares, de lyonnois, de galles), ils sont le plus souvent présentés en minuscule (gannes, mares, galles, lyonnois). II s'avère possible, mais beaucoup moins répandu dans l'édition qu'en cas d'élision, que la majuscule porte sur la préposition à la place du nom de lieu (Dirlande).

\subsection{Analyse de la quatrième partie du Roman de Perceforest ( 233 cas)}

\subsubsection{Au niveau textuel ( 2 cas, $0.8 \%$ )}

\begin{tabular}{|l|l|}
\hline $\mathbf{1}$ & \multicolumn{1}{|c|}{$\mathbf{2}$} \\
\hline $\begin{array}{l}\text { + blancs + une ligne blanche }+ \text { Lettrine de } \\
\text { chapitre (1 cas) }: \text { La Vraye et ancienne }\end{array}$ & $\begin{array}{l}\text { blancs }+ \text { (à la ligne) }+ \text { Lettrine de } \\
\text { paragraphe (1 cas) : Tandis que Troÿlus et le }\end{array}$ \\
histoire nous fait mention que $[\ldots](12 \mathrm{~b})$ & roy Lyonnel se devisoient ensemble [...] $(12 \mathrm{c})$ \\
\hline
\end{tabular}

Au vu de ce relevé, on observe que le chapitre II de la quatrième partie de Perceforest est divisé en deux séquences narratives.

Notons que les éléments se trouvant à l'entrée du chapitre (5 lignes de haut) et du paragraphe (4 lignes de haut) sont respectivement le syntagme nominal (La vraye et ancienne histoire) annonçant le début du chapitre, et le strument temporel (Tandis que) servant à jalonner les étapes chronologiques du récit (Buridant, 1980: 20).

\subsubsection{Au niveau inter-propositionnel (160 cas, $68.6 \%$ )}

\begin{tabular}{|c|c|c|c|c|c|c|}
\hline 3 & 4 & 5 & 6 & 7 & 8 & 9 \\
\hline $\begin{array}{l}T+M \\
(2 \text { cas })\end{array}$ & $\begin{array}{c}.+\mathrm{M} \\
(47 \mathrm{cas})\end{array}$ & $\begin{array}{c}:+M \\
(7 \text { cas })\end{array}$ & $\begin{array}{l}/+M \\
(2 \text { cas })\end{array}$ & $\begin{array}{l}\varnothing+M \\
(2 \text { cas })\end{array}$ & $\begin{array}{c}:+m \\
(41 \text { cas })\end{array}$ & $\begin{array}{c}/+\mathbf{m} \\
(59 \text { cas })\end{array}$ \\
\hline
\end{tabular}

\section{Les combinatoires avec majuscule}

La combinatoire 3 annonce le début de la rubrique et le numéro du chapitre :

(36) If Comment le roy Gadiffer se partit du roy Perceforest son frere a moult belle compaignie. (12 b)

(37) I Chapitre .iie. (12 b)

Les combinatoires à majuscule 4,6 et 7 sont capables de marquer le début du discours direct ou l'alternance des locuteurs dans les dialogues, ou encore le retour au récit :

(38) Mais le roy Gadiffer luy dist. Cher frere cueur de roy doit estre estable en toutes vertus et bonnes operations (12 b)

\footnotetext{
13 G. Ouy (1979: 69) énonce que la virgule couchée peut aussi avoir d'autres formes: trait oblique, deux traits parallèles horizontaux ou obliques.
} 
(39) Quant le roy Perceforest entendit son frere : il commença a dire. Cher frere ie vous remercye de vostre tresbonne doctrine [...]/ \& a dieu vous command. Atant retourna le roy Perceforest/ et le roy gadiffer se mist en chemin a grant compaignie (12 b)

(40) Sire chevalier dist Troÿlus veu qu'il vous en est tant bien advenu/ d'autant estes vous tenu d'en faire meilleure chere/ Certes dist le roy Lyonnel/ (12 c)

(41) il eut grant desir de la secourir et ayder : si dist. Damoiselle ie suis prest d'acomplir vostre donné a entendre : et de secourir la pucelle : aumoins si a moy est possible. Sire dist la messagere la vostre bonne mercy. (12 d)

(42). Si vous requiers que nous partions tantost que nous avrons disné [Ø] Et quant les assistens entendirent que le roy au Daulphin avoit promis d'aller vers la pucelle (12 d)

L'absence de signe de ponctuation peut s'expliquer par le fait que la ligne est déjà remplie jusqu'à la bordure : par manque d'espace, on conserve la majuscule en supprimant le signe.

En plus de l'indication du début du discours direct et du changement de locuteur, la combinatoire 4 peut introduire des séquences corrélatives «subordonnéeprincipale ॥:

(43) . Et quant le roy Perceforest veit que le roy Gadiffer son frere s'en vouloit aller/ il commença a plorer (12 b)

(44) . Et quant Lyonnel fut arrivé en sa place/ ses hommes le bien veignerent grandement ( 12 b)

Ces séquences corrélatives "subordonnée-principale" se rencontrent relativement souvent lors du retour au récit :

(45) Ma treshonnoree dame dist la messagere/ ie suis a une pucelle estrangiere qui a grant mestier du conseil du chevalier/ qui est tant bonne entre toutes les pucelles que ie croy/ s'il estoit ycy il ne consentiroit point d'estre celé. Quant le chevalier au Daulphin eut entendu la messagere/ il fut moult desirant sçavoir qui estoit celle (12 c)

(46) . Et luy sembla si elle pouoit parler a vous/ que moult legierement luy acomplyriez son desir: qui la mettra a mort si vous aucunement ne la secourez. Quant le chevalier eut entendu le message de la damoiselle: il eut grant desir de la secourir et ayder (12 d)

On fera remarquer une occurrence qui annonce la fin du chapitre et le début du suivant, à savoir la formule canonique de transition qui est introduite par la combinatoire 4 et terminée par le signe «point » :

(47) . Atant se taist l'histoire d'eulx tous/ \& retoune a parler du roy au Daulphin pour racompter comment il luy print de son entreprinse. (13 a)

Les combinatoires 5 et 6 délimitent majoritairement des propositions commençant par les connecteurs « mais » (5 cas) et « car » (3 cas) :

(48) : Mais puis que ainsi est ie suis bien ioyeuse de la demeure de vostre femme : pour la vertu \& I'honneur que ie sçay en elle. (13 a)

(49) / Mais pour eschever aucuns perilz qui survenir vous pourroient/ ie vous prie que tous les iours vous portiez cestuy anneau en vostre doy (13 a) 
(50) Cher frere ie vous remercy de vostre tresbonne doctrine: Car iamais ne sera heure que mieulx ne m'en soit/ \& a dieu vous command (12 b)

(51) Damoiselle il me semble estre raison que l'on sache qui vous estes: ains que l'on vous dye rien: Car telle le pourroit demander que vous n'en seriez ia advertye. (12 c)

\section{Les combinatoires avec minuscule}

Les combinatoires 8 et 9 sont susceptibles de baliser tous les types de propositions:

(52) Eł quant Lyonnel fut arrivé en sa place/ ses hommes le bien veignerent grandement/ la y en avoit moult de la forest du glac : comme advertis que Lyonnel estoit couronné roy de leurs marches/ qui tel honneur luy firent que plus ne pouoient (12 b)

Ces deux combinatoires délimitent d'une part, des propositions coordonnées non dépendantes perpétuant ou non les sujets de la proposition précédente, et d'autre part des subordonnées coordonnées:

(53) et mesmes la pucelle a qui ie suis qui tant est gentille bonne et belle : et qui si bien est adressee que elle n'a sa pareille/ s'en alla l'autre iour en ung temple du dieu Mercure (12 d)

(54) La vraye et ancienne histoire nous fait mention que aprés que la feste du dieu souverain fut achevee/ et que les princes/ chevaliers/ dames/ damoiselles et pucelles furent departies au congé du roy Pereceforest [...] : le gentil roy Gadffer/ la royne sa compaigne: leurs deux filz Gadiffer \& Nestor/ \& tous ceulx de leur pays : se mirent en point pour convoyer Lyonnel iusques au chastel qu'il avoit nouvellement fondé (12 b)

(55) Et la chose preste le roy lyonnel vint aux fenestres de son palais: et virent de loing que Blanche la royne sa femme chevauchoit au milieu de ses deux suers \& de ses deux freres : si appella Troÿlus et luy dist. (12 b-12 c)

(56) Atant le gentil roy et plusieurs autres monterent a cheval/ et les allerent bien veigner le plus honnorablement qu'ilz peurent (12 c)

3.2.3. Au niveau intra-propositionnel (71 cas, $30.4 \%$ )

\begin{tabular}{|c|c|c|c|c|}
\hline 5 & 8 & 9 & 10 & 11 \\
\hline$:+M$ & $:+\mathrm{m}$ & $/+\mathrm{m}$ & .chiffre romain ordinal. & $/ /$ en fin de ligne \\
$(3 \mathrm{cas})$ & $(11 \mathrm{cas})$ & $(18 \mathrm{cas})$ & $(1 \mathrm{cas})$ & (39) \\
\hline
\end{tabular}

L'imprimé de 1528 a recours aux combinatoires 5, 8 et 9 pour segmenter les syntagmes ou les mots de même nature syntaxique, notamment dans une série énumérative :

(57) les princes/ chevaliers/ dames/ damoiselles et pucelles furent departies au congé du roy Pereceforest (12 b)

(58) Et si avant qu'il y peult entendre aucunement/les officiers/ sergans/ prevostz/ baillifz ne autres (12 b)

(59) : le gentil roy Gadiffer/ la royne sa compaigne : leurs deux filz Gadiffer \& Nestor/ \& tous ceulx de leur pays : se mirent en point pour convoyer Lyonnel (12 b)

(60) et ainsi ensemble chevaucherent une iournee pres du chastel de Lyonnel/ ou Lyonnel : le Tors : Estonné : Troÿlus \& le roy au Daulphin (12 b) 
Il faut signaler que la majuscule, dans la combinatoire 5, assure plutôt une fonction distinctive qu'une fonction démarcative, car, précédés du signe «: mots en question renvoient tous à des noms propres (Estonné, Troÿlus). En outre, tout comme l'imprimé de 1522, l'imprimé de 1528 favorise visiblement l'emploi de la majuscule dans les noms propres (Gadiffer, Perceforest, Nestor, Lyonnel, Escosse, Tantalon) en nuisant à l'usage de la minuscule (gadifer, lyonnel). Cependant, l'imprimé de 1528 hésite à mettre tous les noms de dieux en majuscule par peur de commettre quelque anachronisme, l'histoire se situant à l'époque du roi grec Alexandre où le monothéisme n'existait pas encore. De ce fait, on met les noms de dieux greco-romains en majuscule comme: le dieu Mercure, tandis que le dieu souverain qui fait allusion au dieu des chrétiens est écrit en minuscule.

Les combinatoires 8 et 9 se trouvent parfois devant des syntagmes prépositionnels à fonction de compléments circonstanciels :

(61) ie quiers ung chevalier qui a acomply les desirs aux douze pucelles niepces du vaillant hermite Pergamon: au fort tournoy entre Sydrac ef Tantalon (12 c)

(62) E† quant il l'eut remercyee \& qu'il fut armé il monta a cheval \& s'en alla avecques la messagiere/ au congé de toute la compaignie ( 13 a)

(63) Combien que sur l'esperance de son bon heur commencerent a danser a la salle et tous a faire bonne chere/ reservé 14 la royne Genievre qui avoir ne pouoit aucune lyesse au cueur pour l'absence de son seigneur (13a)

Comme l'imprimé de 1522, l'imprimé de 1528 emploie aussi la combinatoire 10, i.e. les points symétriques, pour encadrer le chiffre romain ordinal (Chapitre .iie.).

En dernier lieu, on relève 39 fois la combinatoire 11 en fin de ligne : cette pratique de la coupure de mots à l'aide de deux traits obliques se rencontre également dans l'imprimé de 1522 (Enveri//té, Si//re).

\subsection{Analyse de Perceval le Galloys (185 cas)}

\subsubsection{Au niveau textuel ( 2 cas, $1 \%$ )}

\begin{tabular}{|l|l|}
\hline 1 & \multicolumn{1}{|c|}{2} \\
\hline $\begin{array}{l}\text { + une ligne blanche + Lettrine (1 cas) } \\
\text { Durant et pendant le Regne du bon \& } \\
\text { triumphant Roy Artus estoit une notable }\end{array}$ & $\begin{array}{l}\text { Or nous recite L'hystoire que comme ledict } \\
\text { Perceval encores jouvenceau avoit de coustume } \\
\text { Dame vesfve tenant son demaine en une } \\
\text { aller visiter par maniere de recreation les } \\
\text { forest }(2 \mathrm{a})\end{array}$ \\
\hline
\end{tabular}

L'imprimé de 1530 a toujours recours à l'association du signe « point » et de la lettrine pour délimiter les unités textuelles. Les deux lettrines occupent 7 lignes de haut. De même que les imprimés de 1522 et de 1528, les struments temporels se trouvent le plus souvent à l'entrée du livre ou du chapitre.

\subsubsection{Au niveau inter-propositionnel ( 116 cas, $62.7 \%$ )}

\footnotetext{
14 Concernant cette construction introduite par le participe passé "reservé ॥, on peut se demander s'il s'agit d'une proposition participiale ou d'un syntagme prépositionnel. Étant donné que le participe passé "reservé » ne s'accorde pas avec le sujet "la roine genievre ", on est en droit de conclure qu'il fonctionne ici comme une préposition invariable. Le glossaire de l'édition de G. Roussineau nous confirme l'emploi prépositionnel de « reservé » (Roussineau, 1987 : 1389).
} 


\begin{tabular}{|c|c|c|c|c|c|c|c|c|}
\hline 3 & 4 & 5 & 6 & 7 & 8 & 9 & 10 & 11 \\
\hline $\begin{array}{l}T+M \\
(2 \text { cas })\end{array}$ & $\begin{array}{l}++M \\
(40 \\
\text { cas) }\end{array}$ & $\begin{array}{c}:+M \\
(2 \text { cas })\end{array}$ & $\begin{array}{l}+\mathrm{M} \\
(3 \text { cas })\end{array}$ & $\begin{array}{l}(\emptyset+M \\
(7 \text { cas })\end{array}$ & $\begin{array}{l}++m \\
(6 \text { cas })\end{array}$ & $\begin{array}{c}:+m \\
(16 \text { cas })\end{array}$ & $\begin{array}{c}I+\mathbf{m} \\
(40 \text { cas })\end{array}$ & $\begin{array}{l}\text { // en fin de } \\
\text { ligne } \\
(52 \text { cas })\end{array}$ \\
\hline
\end{tabular}

\section{Les combinatoires avec majuscule}

La combinatoire 3 se trouve en général au commencement du livre, du chapitre indiquant le titre du livre, ou du résumé du chapitre :

(64) I Cy commence L'hystoire Recreative contenant les faictz \& gestes du Trespreulx \& vaillant Perceval le Galoyos/ Chevalier de la Table ronde. (2 a)

(65) I Comment et en quelle maniere Perceval eust premiere congnoissance de Chevalerie. E† comment il trouva cinq chevaliers errant en la Gaste forest/ \& du parlement qu'il eust avec eulx. (2 b)

Il convient de souligner que la combinatoire 4 se rencontre assez régulièrement devant des séquences ayant un sens plus ou moins complet :

(66). Ung jour mist bride et celle sur son Chasseron qui est a dire le petit cheval sur lequel il alloit a la chasse des Bestes et Oyseaulx. (2 b)

(67) . Petit aprés veit issir les cinq chevalliers hors du bois et puis quant les apperceut a descouvert ainsy armes l'escu au col et la lance au poing fut plus esmerveille quoncques n'avoit esté car en son vivant chevallier $n$ 'avoit veu ne de chevallier n'avoit ouy parler. (2 c)

Notons également que ces séquences débutent souvent par une proposition subordonnée temporelle en sujet nominal ou pronominal, suivie de propositions régies par le même sujet:

(68) . Quant il fut en la forest entré/ regardant les tans beaulx arbres verdoier \& fleurir/ oyant les gentes gorgettes des oyselletz recreativement armoniser/ fut si tres lyé et joyeulx qu'il ne sçavoit que devenir. (2 b)

(69). Quant les quattre aultres chevalliers eurent long temps attendu et regardé leur maistre au jouvenceau parler/ se prindrent a approcher puis ont dit. (3 a)

D'ailleurs, les combinatoires $4,5,6$ et 7 sont aptes à signaler le passage de la narration au discours direct, le retour au récit, l'alternance des locuteurs dans la conversation, ou le monologue de Perceval :

(70) Quant les quattre aultres chevalliers eurent long temps attendu et regardé leur maistre au jouvenceau parler/ se prindrent a approcher puis ont dit. Certainnement advis nous est que cestui aqui vous parlez n'est pas de grant sçavoir ( 3 a)

(71) Quant il les eust bien regardez lors dist qu'il avoit fort vers dieu mesprins d'avoir proposé que c'estoient diables/ car bien voioit que c'estoient anges/ Certes dist il ma mere m'a tousjours assez adverti dont bien me souvient que les anges sont les plus belles creatures de ce monde aprés dieu qui les a formees. (2 c-2 d)

(72) . respondirent bouviers a leur maistre [Ø] Le long du jour n'ont cessé d'errer et traverser par ses destrois: La responce des bouviers du jouvenceau ouye la raconta aux chevalliers/ puis humblement leur demanda ou le Roy Artus habitoit et se tenoit le plus continuellement ( 3 c-3 d) 
(73) Or dictes moy dist le jouvenceau au chevallier nasquistes vous tel que vous estes: Respond le chevallier que non et que cela ne pourroit estre pareillement que nulle chose ne sçavroit ainsy naistre. (3 b)

(74) Je vous prie dit le jouvenceau prenant le chevallier par son haubert dictes moy que c'est que vous avés vestu. Soiés certain dit le chevalier que c'est ung haubert le quel est faict d'acier et aussy pesant comme fer. Et de quoy vous sert il dit jouvenceau. Je te dis respond le chevallier que il me sert de telle chose que se tu voullois jecter contre moy tous tex javellotz qu'ilz ne me sçavroient grever n'autre chose pareillement [...] (3 b)

(75) / Respond le chevallier mon amy que me le demandés sachés pour vray que de puis cinq jours ce harnois et armures m'ont esté donnees du Roy Artus [...] (3b- $3 c)$

(76) [Ø] Vraiement se dit le jouvenceau ce ne seroit pas bien propice pour les biches ne pour les cerfz: parquoy je prie a dieu les voulloir garder de vestir tel abillement car ainsy ne sçavroient profiter mes javellotz quant occire je les vouldroie et par ainsy jamais aprés eux ne courroie. (3 b)

\section{Les combinatoires avec minuscule}

Les combinatoires 8,9 et 10 délimitent plus souvent les propositions introduites par le sujet nominal ou les divers struments (mais, car, puis, lors) :

(77) . le chevallier luy demdande de rechief s'il n'avoit point veu passer en ceste forest les cinq chevalliers et les tois pucelles/ $(2 \mathrm{~d})$

(78) : le jouvenceau aprochant le chevallier luy prend le bas de son escu en requerant luy dire que c'est qu'il porte ainsy pendu a son col (3 a)

(79) . mais si elle pouoit le desiroit garder pour estre le baston de sa viellesse \& guide de ses affaires ( 2 a)

(80) / mais on en fiert les hommes d'armes et les aultres contre lesquelz on a bataille. ( 3 a)

(81) : car en mon vivant ne veis si belles personnes. (2 d)

(82) / car ilz chevauchoient en grande impetuosité et a grant vistesse \& haste (2 c)

(83) : puis luy souvient que sa mere luy avoit dict aussy que quant on voit les diables qu'il se fault munis du signe de la croix/ lors dist a parsoy que ja ne se signera d'icelle s'il en peult aulcun rencontrer (2 c)

(84) . puis se retournant vers le jouvenceau l'interroga de rechef s'il n'avoit point veu passer par ceste voie les cinq chevalliers et les trois pucelles. (3 b)

(85) : lors le maistre des chevalliers le print a interroger. (2 d)

Il arrive sporadiquement que les combinatoires 8,9 et 10 balisent les répliques des personnages:

(86) Certainement advis nous est que cestui aqui vous parlez n'est pas de grant sçavoir mais assez petitement introduit: vrayement respond le chevallier vous dictes la verité/ ( 3 a)

(87) Or dis moy doncques je te prie que sont devenus si tu les scez les cina chevalliers et les trois pucelles vont ilz le pas ou si s'ensuivent / voiez vous dit 
le jouvenceau au Chevallier ses grans bois qui environnent ceste montaigne/ (3c)

(88) . la sont dit le jouvenceau les laboureurs \& mestoiers de ma mere l'ung y seme ef l'autre y herse ef l'autre y meine la charrue/ (3 c)

Comme l'a constaté C. Marchello-Nizia (2007: 297), on repère parfois la combinatoire 9 dans la construction de la double négation ou devant la deuxième proposition complétive précédée du coordonnant « et»:

(89) ladicte dame ne luy osa jamais declarer comment son pere ne ses freres estoient mors: ne que c'estoit de l'ordre de chevalerie/ craignant que a ce ne se voulsist tourner \& induire: et que par tel art exercer elle peust demeurer seulle et sans enfans. (2 a)

On peut voir que la combinatoire 10 délimite à la fois des propositions subordonnées et des propositions non dépendantes:

(90) E† estoit la principalle \& seulle occupation du Jouvenceau/ fors que aucunesfois il s'esbatoit a jecter et darder Javellotz aprés les cerfz et biches \& aprés les oyseaulx (2 a)

(91) voiez vous dit le jouvenceau au Chevallier ses grans bois qui environnent ceste montaigne/ auprés des destrois y a ung beau val \& fertille $(3 \mathrm{c})$

\subsubsection{Au niveau intra-propositionnel (67 cas, $36.2 \%$ )}

\begin{tabular}{|c|c|c|c|}
\hline 4 & $\mathbf{6}$ & $\mathbf{1 0}$ & 11 \\
\hline$+\mathrm{M}(1$ cas $)$ & $/+\mathbf{M}(1$ cas $)$ & $/+\mathbf{m}(13$ cas $)$ & $/ /$ en fin de ligne $(52$ cas $)$ \\
\hline
\end{tabular}

On s'aperçoit que la combinatoire 6 intervient une fois entre deux syntagmes nominaux dont le deuxième marque l'apposition au premier syntagme. Notons que la majuscule, en l'occurrence, remplit une fonction distinctive et non une fonction démarcative, car la seule occurrence contenant la majuscule à l'initiale du mot (Chevalier) procède d'une pure coïncidence, à savoir la place des noms propres au début du syntagme :

(92) Cy commence L'hystoire Recreative contenant les faictz \& gestes du Trespreulx \& vaillant Perceval le Galoyos/ Chevalier de la Table ronde.

Les combinatoires 4 et 10 se rencontrent de manière éparse entre les syntagmes binômes :

(93) . Et pour ce esbastre \& prendre recreation gettoit ses Javellotz l'ung aprés l'aultre : puis tantost bas/ puis tantost hault/ l'ung a travers/ l'autre a cousté. Puis en avant/ puis en arriere (2 b)

La combinatoire 10 peut aussi scander des syntagmes prépositionnels :

(94) Durant et pendant le Regne du bon \& triumphant Roy Artus estoit une notable Dame vesfve tenant son demaine en une forest nommee en celuy temps/ la gaste forest/ au pais de Galles (2 a)

Si on se fie à l'hypothèse de G. Ouy (1979: 69), l'usage des doubles traits obliques pour la coupure de mots était très ancien. Cette pratique persiste encore dans les premiers imprimés, à telle enseigne que l'imprimé de 1530 nous en offre 52 occurrences: 
(95) il soulloit le plus souvent faire sa demeure a Car//dueil : et que puis peu de temps luy a//voient laissé ( $3 \mathrm{~d}$ )

Pour finir, sauf en de rares cas, l'imprimé de 1530 se préoccupe de distinguer les noms propres des noms communs par la graphie (Perceval le Galoyos, Galles, Gaste forest, Roy Artus, Cardueil) ; cependant, l'imprimeur a tendance à mettre certains noms communs en majuscule, surtout les noms liés à la chevalerie (Chevalliers, Chevalerie), et à la vie paysanne du jeune protagoniste (Laboureurs, Bestes, Oyseaulx, Javellotz). On observe aussi qu'en cas d'élision, avant l'usage systématique de l'apostrophe, la majuscule porte toujours sur l'article défini au lieu de porter sur le nom (Lhystoire).

\section{Conclusion}

L'étude systématique des valeurs des signes combinatoires a permis de dégager certaines tendances homogènes et hétérogènes. À première vue, les trois imprimés de Galliot du Pré disposent tous de onze combinatoires. Cependant, si on les examine de plus près, on se rend compte qu'il subsiste une légère différence entre les trois textes. On remarque l'absence de la combinatoire "signe zéro + Majuscule » dans l'imprimé de 1522, et celle de la combinatoire "point + minuscule " dans l'imprimé de $1528^{15}$. Quant à l'imprimé de 1530, il est privé de points symétriques pour encadrer les chiffres romains.

Du point de vue des combinaisons de signes, les trois imprimés de Galliot du Pré présentent presque la même gamme de signes, à savoir cinq signes de base : le pied-de mouche, le point, le comma, la barre oblique, ainsi que les doubles traits obliques. La pratique de la ponctuation de Galliot du Pré réside dans l'emploi exclusif des signes simples s'associant ou non avec des initiales, des majuscules et des blancs pour former des combinatoires nouvelles.

Au niveau textuel, les trois imprimés privilégient unanimement la combinatoire "point " associée à des blancs ou à une ligne blanche et à une lettrine pour marquer le passage à un nouveau chapitre ou à un nouveau paragraphe.

Au niveau inter-propositionnel, on constate une hiérarchie assez logique dans les trois imprimés: les combinatoires contenant le signe "pied-de-mouche " suivi de la majuscule sont les plus fortes"16; les combinatoires contenant le signe "barre oblique " suivi de la minuscule, les plus faibles ${ }^{17}$. La preuve en est que la combinatoire "point + Majuscule » introduit fréquemment des séquences de propositions syntaxiquement autonomes, ayant un sens plus ou moins complet (ex. 9, 10, 43-47, 66-69). Après elles, les combinatoires contenant le signe « comma » associé à la majuscule ponctuent le plus souvent des propositions non dépendantes, de moindre étendue (ex. 5, 50, 51), ou des séquences de propositions composées "subordonnée-principale " appuyées ou non par le strument "mais» (ex. 48, 72). Notons que les combinatoires «point + Majuscule ", " barre oblique + Majuscule » et

\footnotetext{
15 Dans notre thèse (2007 : 540), l'imprimé de 1528 nous offre une fois le point d'interrogation et trois fois les parenthèses : malheureusement, ces deux signes ne figurent pas dans le corpus sélectionné.

16 La combinatoire "pied-de-mouche + Majuscule " participe à la mise en page du texte, car elle marque le début de la rubrique et le numéro du chapitre (ex: 1, 2, 33, 34, 60, 61).

17 À notre sens, la ponctuation forte délimite en général des unités syntaxiquement autonomes, ayant un sens plus ou moins complet, alors que la ponctuation faible sépare plutôt des unités incomplètes du point de vue syntaxique comme de celui du sens.
} 
« signe zéro + Majuscule » signalent très régulièrement le début du discours direct, le changement de locuteur ou le retour à la narration (ex. 11-14, 38-42, 70-71, 74-76). Cependant, il est possible que les répliques des personnages soient marquées par les combinatoires contenant une minuscule, soit: "comma + minuscule"), "barre oblique + minuscule » et " point + minuscule » (ex. : 86-88). En ce qui concerne la combinatoire «barre oblique + minuscule », elle balise tous les types de propositions, le plus couramment les propositions commençant par les connecteurs « car, mais, et $॥$ (ex. 24, 54, 56, 80, 82), et les relatives (ex. 25-27), ainsi que les principales postposées aux subordonnées (ex. 9, 20, 22, 43, 44, 68, 69). II semble que la combinatoire " comma + minuscule » concurrence la combinatoire « barre oblique + minuscule "), du fait qu'elle peut délimiter non seulement les propositions introduites par un sujet nominal ou des struments d'ordre divers (ex. 15-17, 78, 81, 83, 85), mais aussi les subordonnées coordonnées (ex. 53, 89).

Au niveau intra-propositionnel, la combinatoire "barre oblique + minuscule» s'impose communément dans les trois imprimés. Elle s'utilise ordinairement dans une kyrielle énumérative (ex. 28, 29, 57, 58, 93). Pourtant, elle se trouve occasionnellement devant des syntagmes prépositionnels à valeur de compléments circonstanciels (ex. 30, 31, 62, 63, 94), et à la frontière des syntagmes binômes reliés ou non par le coordonnant "et» (ex. 59, 93). N'oublions pas que la combinatoire "barre oblique + Majuscule " a parfois pour fonction de mettre l'accent sur le syntagme (ex. 60). De même que la combinatoire "barre oblique + minuscule ", la combinatoire "comma + minuscule" ponctue quelquefois les syntagmes en cas d'énumération (ex. : 28, 59, 60). II convient de rappeler que les points symétriques entourant les chiffres romains s'utilisent régulièrement dans les imprimés de Galliot du Pré pour éviter la confusion entre les lettres et les chiffres (Ex. 32, 33, 37). Au niveau des mots, les doubles traits obliques sont usités dans les trois imprimés pour avertir le lecteur que le dernier mot en fin de ligne se continue à la page suivante (ex: 34 , $35,95)$. Cependant, l'emploi de ce signe n'est pas obligatoire, comme on peut l'observer dans notre corpus.

Enfin, l'usage de la majuscule pour les noms propres s'avère très fréquent dans les trois imprimés. Mais dans l'imprimé de 1530, Galliot du Pré a tendance à mettre en majuscule les noms communs ayant trait à la chevalerie (Chevalliers, Chevalerie) et à la vie paysanne de Perceval (Laboureurs, Bestes, Oyseaulx, Javellotz).

Cette étude, pour le moment limitée à quelques folios de trois imprimés du début du XVIe siècle, nous permet cependant de conclure provisoirement que le système de ponctuation de Galliot du Pré est assez systématique et hiérarchisé. II semble que Galliot du Pré connaisse bien les traités médiévaux et ceux de son époque, car les formes des signes employées par l'éditeur humaniste se rencontrent de manière très répandue dans les manuscrits médiévaux, à part celle du comma (:). Or nous remarquons que les valeurs du comma basculent entre la ponctuation moyenne et la ponctuation faible, étant donné que la barre oblique et le comma, dans certaines constructions syntaxiques, sont interchangeables. Surtout au niveau interpropositionnel, certaines valeurs sont partagées par plusieurs combinatoires. Cette variabilité des valeurs des signes est-elle liée à la confusion dans la terminologie ? II faudrait élargir encore le corpus afin de confirmer notre hypothèse dans une prochaine recherche. 


\section{Bibliographie}

ANDRIEUX-REIX, N. et MONSONÉGO, S. (1997) : "Écrire des phrases au Moyen Âge, matériaux et premières réflexions pour une étude des segments graphiques observés dans des manuscrits français médiévaux ॥. Romania, n¹15, p. 289336.

BELTRAN, É (1985) : "Un traité inconnu de Guillaume Fichet sur la ponctuation». Scriptorium, Tome 39, $n^{\circ}$ 2, p. 284-291.

BURIDANT, C. (1980) : "Le strument et et ses rapports avec la ponctuation dans quelques textes médiévaux ॥. Théories linguistiques et traditions grammaticales. A. M. DESSAUX-BERTHONNEAU (éd.). Lille : Presses universitaires de Lille, p. 13-53.

CATACH, N. (1968) : L'orthographe française à l'époque de la Renaissance, AuteursImprimeurs-Ateliers d'imprimerie. Genève : Droz.

- (1988) : «Retour aux sources ». Traverses, Le génie de la ponctuation, n 43. p.3347.

— (1994) : La Ponctuation. Paris : PUF. Coll. « Que sais-je ? » n² 2818.

CHARON-PARENT, A. (1988) : "Aspects de la politique éditoriale de Galliot du Pré ». Le Livre dans l'Europe de la Renaissance. Actes du XXVIIII colloque international d'études humanistes de Tours. Promodis : Éditions du Cercle de la Librairie, p. 209-218

DELALAIN, P. (1890) : Notice sur Galliot du Pré. Libraire parisien de 1512 à 1560. Paris : Cercle de la Librairie.

DOPPAGNE, A. (1991): Majuscule, abréviations, symboles et sigles, pour une toilette parfaite du texte. Bruxelles : Duculot.

HUBERT, M. (1972) : "Le vocabulaire de la "ponctuation" aux temps médiévaux". Archivum Latinitatis Medii AEvi, 38, p. 57-166.

LAVRENTIEV, A. (2000) : "À propos de la ponctuation dans I'Image du monde ». La Licorne, 52, p. 23-35.

- (2011): "Les changements dans les pratiques de la ponctuation liés au développement de l'imprimerie à la fin du XVe siècle et au début du XVIe siècle ». La ponctuation à la Renaissance. N. DAUVOIS et J. DÜRRENMATT (éds.). Paris : Classiques Garnier, p. 31-56.

- (2016): "Ponctuation française du Moyen Âge au XVIe siècle: théories ef pratiques ॥. In La ponctuation à l'aube du XXe siècle. Perspectives historiques et usages contemporains. Limoges : Lambert Lucas, p. 39-62.

LI, H.-C. (2007) : Découpage et structuration du texte : Lettrines, majuscules, blancs et autres signes de ponctuation dans les versions manuscrites et imprimées du Roman de Perceforest : étude comparative. Thèse de doctorat, Université de Strasbourg.

MARCHELLO-NIZIA, C. (1978 a) : "Ponctuation et "unités de lecture" dans les manuscrits médiévaux ou : je ponctue, tu lis, il théorise ॥. Langue française, 40, p. 32-44.

— (1978 b) : « Un problème de linguistique textuelle : la classe des éléments joncteurs de propositions $»$. Études de syntaxe du moyen français. R. MARTIN (éd.). Paris : Klincksieck, p. 33-40. 
- (2007) : « Le comma dans un manuscrit en prose du 13e siècle : grammaticalisation d'un marqueur de corrélation, ou marquage d'intonation? ॥. Discours, diachronie, stylistique du français. Études en hommage à Bernard Combettes. O. BERTRAND et al. (éds). Berne : Peter Lang, p. 293-305.

OUY, G. (1979) : « La ponctuation des premiers humanistes français ». La ponctuation. Recherches historiques et actuelles (Actes de la Table Ronde Internationale CNRS, mais 1978). N. CATACH et J. PETIT (éds.). †. II. Paris: GTM-CNRS-HESO, p. 56-89.

PARENT, A. (1974) : Les métiers du livre à Paris au XVle siècle (1535-1560). Genève: Droz, p. 217-251.

POIRION, D. (1978) : «Les paragraphes et le pré-texte de Villehardouin ». Langue française, 40, p. 45-59.

ROUSSINEAU, G. (1987) : Perceforest, quatrième partie, t. I et II. Genève : Droz.

TROYES, C. de (1990): Le Roman de Perceval. Édition du ms. 354 de Berne, traduction critique, présentation et notes de Charles Méla. Paris: Librairie générale française (Le livre de poche, 4525. Lettres gothiques).

VAN HEMELRYCK, T. (2016) : «Du Perceforest manuscrit à l'uimprimé de Galliot du Pré (1528): un long fleuve tranquille? ». Le Roman français dans les premiers imprimés. A. SCHOYSMAN et M. COLOMBO-TOMELLI (dir.). Paris: Classiques Garnier, p. 159-174.

ZINK, G. (1990) : Le moyen français. Paris : PUF. Coll. « Que sais-je ? » nº 1086. 